\title{
Oscillation Criteria for Second-Order Neutral Delay Dynamic Equations with Mixed Nonlinearities
}

\author{
Ethiraju Thandapani, ${ }^{1}$ Veeraraghavan Piramanantham, ${ }^{2}$ \\ and Sandra Pinelas ${ }^{3}$ \\ ${ }^{1}$ Ramanujan Institute for Advanced Study in Mathematics, University of Madras, Chennai 600 005, India \\ ${ }^{2}$ Department of Mathematics, Bharathidasan University, Tiruchirappalli 620 024, India \\ ${ }^{3}$ Departamento de Matemática, Universidade dos Açores, 9501-801 Ponta Delgada, Azores, Portugal
}

Correspondence should be addressed to Sandra Pinelas, sandra.pinelas@clix.pt

Received 20 September 2010; Revised 30 November 2010; Accepted 23 January 2011

Academic Editor: Istvan Gyori

Copyright (C) 2011 Ethiraju Thandapani et al. This is an open access article distributed under the Creative Commons Attribution License, which permits unrestricted use, distribution, and reproduction in any medium, provided the original work is properly cited.

This paper is concerned with some oscillation criteria for the second order neutral delay dynamic equations with mixed nonlinearities of the form $(r(t) u(t))^{\Delta}+q(t)|x(\tau(t))|^{\alpha-1} x(\tau(t))+$ $\sum_{i=1}^{n} q_{i}(t)\left|x\left(\tau_{i}(t)\right)\right|^{\alpha_{i}-1} x\left(\tau_{i}(t)\right)=0$, where $t \in \mathbb{T}$ and $u(t)=\left|(x(t)+p(t) x(\delta(t)))^{\Delta}\right|^{\alpha-1}(x(t)+$ $p(t) x(\delta(t)))^{\Delta}$ with $\alpha_{1}>\alpha_{2}>\cdots>\alpha_{m}>\alpha>\alpha_{m+1}>\cdots>\alpha_{n}>0$. Further the results obtained here generalize and complement to the results obtained by Han et al. (2010). Examples are provided to illustrate the results.

\section{Introduction}

Since the introduction of time scale calculus by Stefan Hilger in 1988, there has been great interest in studying the qualitative behavior of dynamic equations on time scales, see, for example, [1-3] and the references cited therein. In the last few years, the research activity concerning the oscillation and nonoscillation of solutions of ordinary and neutral dynamic equations on time scales has been received considerable attention, see, for example, [4-8] and the references cited therein. Moreover the oscillatory behavior of solutions of second order differential and dynamic equations with mixed nonlinearities is discussed in [9-16].

In 2004, Agarwal et al. [5] have obtained some sufficient conditions for the oscillation of all solutions of the second order nonlinear neutral delay dynamic equation

$$
\left(r(t)\left((y(t)+p(t) y(t-\tau))^{\Delta}\right)^{r}\right)^{\Delta}+f(t, y(t-\delta))=0
$$


on time scale $\mathbb{T}$, where $t \in \mathbb{T}, \gamma$ is a quotient of odd positive integers such that $\gamma \geq 1, r(t)$, $p(t)$ are real valued rd-continuous functions defined on $\mathbb{T}$ such that $r(t)>0,0 \leq p(t)<1$, and $f(t, u) \geq q(t)|u|^{\gamma}$.

In 2009, Tripathy [17] has considered the nonlinear neutral dynamic equation of the form

$$
\left(r(t)\left((y(t)+p(t) y(t-\tau))^{\Delta}\right)^{\gamma}\right)^{\Delta}+q(t)|y(t-\delta)|^{\gamma} \operatorname{sgn} y(t-\delta)=0, \quad t \in \mathbb{T},
$$

where $\gamma>0$ is a quotient of odd positive integers, $r(t), q(t)$ are positive real valued rdcontinuous functions on $\mathbb{T}, p(t)$ is a nonnegative real valued rd-continuous function on $\mathbb{T}$ and established sufficient conditions for the oscillation of all solutions of (1.2) using Ricatti transformation.

Saker et al. [18], Şahíner [19], and Wu et al. [20] established various oscillation results for the second order neutral delay dynamic equations of the form

$$
\left(r(t)\left((y(t)+p(t) y(\tau(t)))^{\Delta}\right)^{r}\right)^{\Delta}+f(t, y(\delta(t)))=0, \quad t \in \mathbb{T}
$$

where $0 \leq p(t)<1, \gamma \geq 1$ is a quotient of odd positive integers, $r(t), p(t)$ are real valued nonnegative rd-continuous functions on $\mathbb{T}$ such that $r(t)>0$, and $f(t, u) \geq q(t)|u|^{\gamma}$.

In 2010, Sun et al. [21] are concerned with oscillation behavior of the second order quasilinear neutral delay dynamic equations of the form

$$
\left(r(t)\left(z^{\Delta}(t)\right)^{\gamma}\right)^{\Delta}+q_{1}(t) x^{\alpha}\left(\tau_{1}(t)\right)+q_{2}(t) x^{\beta}\left(\tau_{2}(t)\right)=0, \quad t \in \mathbb{T},
$$

where $z(t)=x(t)+p(t) x\left(\tau_{0}(t)\right), \gamma, \alpha, \beta$ are quotients of odd positive integers such that $0<\alpha<$ $\gamma<\beta$ and $\gamma \geq 1, r(t), p(t), q_{1}(t)$, and $q_{2}(t)$ are real valued rd-continuous functions on $\mathbb{T}$.

Very recently, Han et al. [22] have established some oscillation criteria for quasilinear neutral delay dynamic equation

$$
\left(r(t)\left|x^{\Delta}(t)\right|^{\gamma-1} x^{\Delta}\right)^{\Delta}+q_{1}(t)\left|y\left(\delta_{1}(t)\right)\right|^{\alpha-1} y\left(\delta_{1}(t)\right)+q_{2}(t)\left|y\left(\delta_{2}(t)\right)\right|^{\beta-1} y\left(\delta_{2}(t)\right)=0, \quad t \in \mathbb{T},
$$

where $x(t)=y(t)+p(t) y(\tau(t)), \alpha, \beta, \gamma$ are quotients of odd positive integers such that $0<\alpha<$ $\gamma<\beta, r(t), p(t), q_{1}(t)$, and $q_{2}(t)$ are real valued rd-continuous functions on $\mathbb{T}$.

Motivated by the above observation, in this paper we consider the following second order neutral delay dynamic equation with mixed nonlinearities of the form:

$$
(r(t) u(t))^{\Delta}+q(t)|x(\tau(t))|^{\alpha-1} x(\tau(t))+\sum_{i=1}^{n} q_{i}(t)\left|x\left(\tau_{i}(t)\right)\right|^{\alpha_{i}-1} x\left(\tau_{i}(t)\right)=0,
$$

where $\mathbb{T}$ is a time scale, $t \in \mathbb{T}$ and $u(t)=\left|(x(t)+p(t) x(\delta(t)))^{\Delta}\right|^{\alpha-1}(x(t)+p(t) x(\delta(t)))^{\Delta}$, and this includes all the equations (1.1)-(1.5) as special cases. 
By a proper solution of (1.6) on $\left[t_{0}, \infty\right)_{\mathbb{T}}$ we mean a function $x(t) \in C_{\mathrm{rd}}^{1}\left[t_{0}, \infty\right)$, which has a property that $r(t)(x(t)+p(t) x(\tau(t)))^{\alpha} \in C_{\mathrm{rd}}^{1}\left[t_{0}, \infty\right)$, and satisfies $(1.6)$ on $\left[t_{x}, \infty\right)_{\mathbb{T}}$. For the existence and uniqueness of solutions of the equations of the form (1.6), refer to the monograph [2]. As usual, we define a proper solution of (1.6) which is said to be oscillatory if it is neither eventually positive nor eventually negative. Otherwise it is known as nonoscillatory.

Throughout the paper, we assume the following conditions:

$\left(C_{1}\right)$ the functions $\delta, \tau, \tau_{i}: \mathbb{T} \rightarrow \mathbb{T}$ are nondecreasing right-dense continuous and satisfy $\delta(t) \leq t, \tau(t) \leq t, \tau_{i}(t) \leq t$ with $\lim _{t \rightarrow \infty} \delta(t)=\infty, \lim _{t \rightarrow \infty} \tau(t)=\infty$, and $\lim _{t \rightarrow \infty} \tau_{i}(t)=$ $\infty$ for $i=1,2, \ldots, n$;

$\left(C_{2}\right) p(t)$ is a nonnegative real valued rd-continuous function on $\mathbb{T}$ such that $0 \leq p(t)<1$;

$\left(C_{3}\right) r(t), q(t)$ and $q_{i}(t), i=1,2, \ldots, n$ are positive real valued rd-continuous functions on $\mathbb{T}$ with $r^{\Delta}(t) \geq 0$;

$\left(C_{4}\right) \alpha, \alpha_{i}, i=1,2, \ldots, n$ are positive constants such that $\alpha_{1}>\alpha_{2}>\cdots>\alpha_{m}>\alpha>\alpha_{m+1}>$ $\cdots>\alpha_{n}>0(n>m \geq 1)$.

We consider the two possibilities

$$
\begin{aligned}
& \int_{t_{0}}^{\infty} \frac{1}{r^{1 / \alpha}(s)} \Delta s=\infty, \\
& \int_{t_{0}}^{\infty} \frac{1}{r^{1 / \alpha}(s)} \Delta s<\infty .
\end{aligned}
$$

Since we are interested in the oscillatory behavior of the solutions of (1.6), we may assume that the time scale $\mathbb{T}$ is not bounded above, that is, we take it as $\left[t_{0}, \infty\right)_{\mathbb{T}}=\left\{t \geq t_{0}: t \in\right.$ $\mathbb{T}\}$.

The paper is organized as follows. In Section 2, we present some oscillation criteria for (1.6) using the averaging technique and the generalized Riccati transformation, and in Section 3, we provide some examples to illustrate the results.

\section{Oscillation Results}

We use the following notations throughout this paper without further mention:

$$
\begin{gathered}
d_{+}(t)=\max \{0, d(t)\}, \quad d_{-}(t)=\max \{0,-d(t)\} \\
Q(t)=q(t)(1-p(\tau(t)))^{\alpha}, \quad Q_{i}(t)=q_{i}(t)\left(1-p\left(\tau_{i}(t)\right)\right)^{\alpha_{i}}, \quad i=1,2,3, \ldots, n, \\
\kappa(t)=\frac{\sigma(t)}{t}, \quad \beta(t)=\frac{\tau(t)}{\sigma(t)}, \quad \beta_{i}(t)=\frac{\tau_{i}(t)}{\sigma(t)}, \quad z(t)=x(t)+p(t) x(\delta(t)) .
\end{gathered}
$$

In this section, we obtain some oscillation criteria for (1.6) using the following lemmas. Lemma 2.1 is an extension of Lemma 1 of [13]. 
Lemma 2.1. Let $\alpha_{i}, i=1,2, \ldots, n$ be positive constants satisfying

$$
\alpha_{1}>\alpha_{2}>\cdots>\alpha_{m}>\alpha>\alpha_{m+1}>\cdots>\alpha_{n}>0 .
$$

Then there is an $n$-tuple $\left(\eta_{1}, \eta_{2}, \ldots, \eta_{n}\right)$ satisfying

$$
\sum_{i=1}^{n} \alpha_{i} \eta_{i}=\alpha
$$

which also satisfies either

$$
\sum_{i=1}^{n} \eta_{i}<1, \quad 0<\eta_{i}<1
$$

or

$$
\sum_{i=1}^{n} \eta_{i}=1, \quad 0<\eta_{i}<1
$$

In the following results we use the Keller's Chain rule [1] given by

$$
\left(y^{\alpha}(t)\right)^{\Delta}=\alpha y^{\Delta}(t) \int_{0}^{1}\left[h y^{\sigma}(t)+(1-h) y(t)\right]^{\alpha-1} d h
$$

where $y$ is a positive and delta differentiable function on $\mathbb{T}$.

Lemma 2.2 (see [23]). Let $f(u)=B u-A u^{(\alpha+1) / \alpha}$, where $A>0$ and $B$ are constants, $\gamma$ is a positive integer. Then $f$ attains its maximum value on $\mathbb{R}$ at $u *=\left(B^{\gamma} / A^{\gamma+1}\right)^{\gamma}$, and

$$
\max _{u \in \mathbb{R}} f=f(u *)=\frac{\gamma^{\gamma}}{(\gamma+1)^{(\gamma+1)}} \frac{B^{\gamma+1}}{A^{\gamma}} .
$$

Lemma 2.3. Assume that (1.7) holds. If $x(t)$ is an eventually positive solution of (1.6), then there exists a $T \in\left[t_{0}, \infty\right)_{\mathbb{T}}$ such that $z(t)>0, z^{\Delta}(t)>0$, and $\left(r(t)\left(z^{\Delta}(t)\right)^{\alpha}\right)^{\Delta}<0$ for $t \in[T, \infty)_{\mathbb{T}}$. Moreover one obtains

$$
x(t) \geq(1-p(t)) z(t), \quad t \geq t_{1} .
$$

Since the proof of Lemma 2.3 is similar to that of Lemma 2.1 in [6], we omit the details.

Lemma 2.4. Assume that (1.7) and

$$
\int_{t_{0}}^{\infty} \tau^{\alpha}(s) Q(s) \Delta s=\infty
$$


hold. If $x(t)$ is an eventually positive solution of (1.6), then

$$
z^{\Delta \Delta}(t)<0, \quad z(t) \geq t z^{\Delta}(t)
$$

and $z(t) / t$ is strictly decreasing.

Proof. From Lemma 2.3, we have $\left(r(t)\left(z^{\Delta}(t)\right)^{\alpha}\right)^{\Delta}<0$ and

$$
\left(r(t)\left(z^{\Delta}(t)\right)^{\alpha}\right)^{\Delta}=r^{\Delta}(t)\left(z^{\Delta}(t)\right)^{\alpha}+r(\sigma(t))\left(\left(z^{\Delta}(t)\right)^{\alpha}\right)^{\Delta}
$$

Since $r^{\Delta}(t) \geq 0$, we have $\left(\left(z^{\Delta}(t)\right)^{\alpha}\right)^{\Delta}<0$. Now using the Keller's Chain rule, we find that

$$
0<\left(\left(z^{\Delta}(t)\right)^{\alpha}\right)^{\Delta}=\alpha z^{\Delta \Delta}(t) \int_{0}^{1}\left(h z^{\Delta}(t)+(1-h) z^{\Delta}(t)\right)^{\alpha-1} d h
$$

or $z^{\Delta \Delta}(t)<0$. Let $Z(t):=z(t)-t z^{\Delta}(t)$. Clearly $Z^{\Delta}(t)=-\sigma(t) z^{\Delta \Delta}(t)>0$. We claim that there is a $t_{1} \in\left[t_{0}, \infty\right)_{\mathbb{T}}$ such that $Z(t)>0$ on $\left[t_{1}, \infty\right)_{\mathbb{T}}$. Assume the contrary, then $Z(t)<0$ on $\left[t_{1}, \infty\right)_{\mathbb{T}}$. Therefore,

$$
\left(\frac{z(t)}{t}\right)^{\Delta}=\frac{t z^{\Delta}(t)-z(t)}{t \sigma(t)}=-\frac{Z(t)}{t \sigma(t)}>0, \quad t \in\left[t_{1}, \infty\right)_{\mathbb{T}}
$$

which implies that $z(t) / t$ is strictly increasing on $\left[t_{1}, \infty\right)_{\mathbb{T}}$. Pick $t_{2} \in\left[t_{1}, \infty\right)_{\mathbb{T}}$ so that $\tau(t) \geq \tau\left(t_{2}\right)$ and $\tau_{i}(t) \geq \tau_{i}\left(t_{2}\right)$ for $t \geq t_{2}$. Then $z(\tau(t)) / \tau(t) \geq z\left(\tau\left(t_{2}\right)\right) / \tau\left(t_{2}\right)=: d>0$, and $z(\tau(t)) / \tau(t) \geq$ $z\left(\tau\left(t_{2}\right)\right) / \tau\left(t_{2}\right)=: d_{i}>0$, so that $z(\tau(t))>\tau(t)$ for $t \geq t_{2}$.

Using the inequality (2.8) in (1.6), we have that

$$
\left(r(t)\left(z^{\Delta}(t)\right)^{\alpha}\right)^{\Delta}+Q(t) z^{\alpha}(\tau(t))+\sum_{i=1}^{n} Q_{i}(t) z^{\alpha_{i}}\left(\tau_{i}(t)\right) \leq 0 .
$$

Now by integrating from $t_{2}$ to $t$, we have

$$
r(t)\left(z^{\Delta}(t)\right)^{\alpha}-r\left(t_{2}\right)\left(z^{\Delta}\left(t_{2}\right)\right)^{\alpha}+\int_{t_{2}}^{t}\left[Q(s) z^{\alpha}(\tau(s))+\sum_{i=1}^{n} Q_{i}(s) z^{\alpha_{i}}\left(\tau_{i}(s)\right)\right] \Delta s \leq 0
$$

which implies that

$$
\begin{aligned}
r\left(t_{2}\right) z^{\Delta}\left(t_{2}\right) & \geq r(t) z^{\Delta}(t)+\int_{t_{2}}^{t}\left[Q(s) z^{\alpha}(\tau(s))+\sum_{i=1}^{n} Q_{i}(s) z^{\alpha_{i}}\left(\tau_{i}(s)\right)\right] \Delta s \\
& >d^{\alpha} \int_{t_{2}}^{t} Q(s) \tau^{\alpha}(s) \Delta s+\sum_{i=1}^{n} d_{i}^{\alpha_{i}} \int_{t_{2}}^{t} Q_{i}(s) \tau_{i}^{\alpha_{i}}(s) \Delta s
\end{aligned}
$$


which contradicts $(2.4)$. Hence there is a $t_{1} \in\left[t_{0}, \infty\right)_{\mathbb{T}}$ such that $Z(t)>0$ on $\left[t_{1}, \infty\right)_{\mathbb{T}}$. Consequently,

$$
\left(\frac{z(t)}{t}\right)^{\Delta}=\frac{t z^{\Delta}(t)-z(t)}{t \sigma(t)}=-\frac{Z(t)}{t \sigma(t)}<0, \quad t \in\left[t_{1}, \infty\right)_{\mathbb{T}}
$$

and we have that $z(t) / t$ is strictly decreasing on $\left[t_{1}, \infty\right)_{\mathbb{T}}$.

Theorem 2.5. Assume that condition (1.7) holds. Let $\left(\eta_{1}, \eta_{2}, \ldots, \eta_{n}\right)$ be $n$-tuple satisfying (2.3) of Lemma 2.1. Furthermore one assumes that there exist positive delta differentiable function $\rho(t)$ and $a$ nonnegative delta differentiable function $\phi(t)$ such that

$$
\limsup _{t \rightarrow \infty} \int_{t_{1}}^{t} \rho(\sigma(s))\left[Q^{*}(s)-\phi^{\Delta}(s)-\frac{\left(\rho^{\Delta}(s)\right)_{+}}{\rho^{\sigma}(s)} \phi(s)-\frac{\kappa^{\alpha^{2}}(s)}{(\alpha+1)^{\alpha+1}} \frac{r(s)\left(\rho^{\Delta}(s)\right)_{+}^{\alpha+1}}{(\rho(\sigma(s)))^{\alpha+1}}\right] \Delta s=\infty,
$$

for all sufficiently large $t_{1}$ where $Q^{*}(t)=Q(t) \beta^{\alpha}(t)+\eta \prod_{i=1}^{n} Q_{i}^{\eta_{i}}(t) \beta_{i}^{\alpha_{i} \eta_{i}}(t)$, and $\eta=\prod_{i=1}^{n} \eta_{i}^{-\eta_{i}}$. Then every solution of (1.6) is oscillatory.

Proof. Suppose that there is a nonoscillatory solution $x(t)$ of (1.6). We assume that $x(t)$ is an eventually positive for $t \geq t_{0}$ (since the proof for the case $x(t)<0$ eventually is similar). From the definition of $z(t)$ and Lemma 2.3, there exists $t_{1} \geq t_{0}$ such that, for $t \geq t_{1}$,

$$
z(t)>0, \quad z(\delta(t))>0, \quad z(\tau(t))>0, \quad z\left(\tau_{i}(t)\right)>0, \quad z^{\Delta}(t)>0, \quad\left(r(t)\left(z^{\Delta}(t)\right)^{\alpha}\right)^{\Delta} \leq 0 .
$$

Define

$$
w(t)=\rho(t)\left[\frac{r(t)\left(z^{\Delta}(t)\right)^{\alpha}}{z^{\alpha}(t)}+\phi(t)\right], \quad t \geq t_{1}
$$

Then from (2.19), we have $w(t)>0$ and

$$
\begin{aligned}
w^{\Delta}(t)= & \frac{\rho^{\Delta}(t)}{\rho(t)} w(t)+\rho(\sigma(t))\left[\frac{r(t)\left(z^{\Delta}(t)\right)^{\alpha}}{z^{\alpha}(t)}\right]^{\Delta}+\rho(\sigma(t)) \phi^{\Delta}(t) \\
\leq & \frac{\rho^{\Delta}(t)}{\rho(t)} w(t)+\rho(\sigma(t)) \frac{\left(r(t)\left(z^{\Delta}(t)\right)^{\alpha}\right)^{\Delta}}{z^{\alpha}(\sigma(t))} \\
& -\rho(\sigma(t)) \frac{r(t)\left(z^{\Delta}(t)\right)^{\alpha}\left(z^{\alpha}(t)\right)^{\Delta}}{z^{\alpha}(t) z^{\alpha}(\sigma(t))}+\rho(\sigma(t)) \phi^{\Delta}(t) .
\end{aligned}
$$


From Keller's chain rule, we have, from Lemma 2.1,

$$
\left(z^{\alpha}(t)\right)^{\Delta} \geq \begin{cases}\alpha z^{\alpha-1}(t) z^{\Delta}(t), & \alpha \geq 1 \\ \alpha z^{\alpha-1}(\sigma(t)) z^{\Delta}(t), & 0<\alpha<1\end{cases}
$$

Using (2.22) and the definition of $\kappa(t)$ in (2.21), we obtain

$$
\begin{aligned}
w^{\Delta}(t) \leq & -\frac{\rho(\sigma(t))}{z^{\alpha}(\sigma(t))}\left[Q(t) z^{\alpha}(\tau(t))+\sum_{i=1}^{n} Q_{i}(t) z^{\alpha_{i}}\left(\tau_{i}(t)\right)\right]+\frac{\rho^{\Delta}(t)}{\rho(t)} w(t) \\
& -\frac{\alpha \rho(\sigma(t))}{r^{1 / \alpha}(t)} \frac{1}{\kappa^{\alpha}(t)}\left|\frac{w(t)}{\rho(t)}-\phi(t)\right|^{(\alpha+1) / \alpha}+\rho(\sigma(t)) \phi^{\Delta}(t) .
\end{aligned}
$$

From Lemma 2.4, we see that $z(t) / t$ is strictly decreasing on $\left[t_{1}, \infty\right)_{\mathbb{T}}$, and therefore

$$
\frac{z\left(\tau_{i}(t)\right)}{\tau_{i}(t)} \geq \frac{z(\sigma(t))}{\sigma(t)}
$$

or

$$
\frac{z\left(\tau_{i}(t)\right)}{z(\sigma(t))} \geq \frac{\tau_{i}(t)}{\sigma(t)}
$$

since $\tau_{i}(t) \leq \sigma(t)$ for all $i=1,2, \ldots, n$. Using (2.25) in (2.23), we have

$$
\begin{aligned}
w^{\Delta}(t) \leq & -\rho(\sigma(t))\left[Q(t) \beta^{\alpha}(t)+\sum_{i=1}^{n} Q_{i}(t) \beta_{i}^{\alpha_{i}}(t) z^{\alpha_{i}-\alpha}(\sigma(t))\right]+\frac{\rho^{\Delta}(t)}{\rho(t)} w(t) \\
& -\frac{\alpha \rho(\sigma(t))}{r^{1 / \alpha}(t)} \frac{1}{\kappa^{\alpha}(t)}\left|\frac{w(t)}{\rho(t)}-\phi(t)\right|^{(\alpha+1) / \alpha}+\rho(\sigma(t)) \phi^{\Delta}(t) .
\end{aligned}
$$

Now let $u_{i}(t)=\left(1 / \eta_{i}\right) Q_{i}(t) \beta_{i}^{\alpha_{i}} z^{\alpha_{i}-\alpha}(\sigma(t)), i=1,2, \ldots, n$. Then (2.26) becomes

$$
\begin{aligned}
w^{\Delta}(t) \leq & -\rho(\sigma(t))\left[Q(t) \beta^{\alpha}(t)+\sum_{i=1}^{n} \eta_{i} u_{i}(t)\right]+\frac{\rho^{\Delta}(t)}{\rho(t)} w(t) \\
& -\frac{\alpha \rho(\sigma(t))}{r^{1 / \alpha}(t)} \frac{1}{\mathcal{K}^{\alpha}(t)}\left|\frac{w(t)}{\rho(t)}-\phi(t)\right|^{(\alpha+1) / \alpha}+\rho(\sigma(t)) \phi^{\Delta}(t) .
\end{aligned}
$$


By Lemma 2.1 and using the arithmetic-geometric inequality $\sum_{i=1}^{n} \eta_{i} u_{i} \geq \prod_{i=1}^{n} \eta_{i}^{u_{i}}$ in (2.27), we obtain

$$
\begin{aligned}
w^{\Delta}(t) \leq & -\rho(\sigma(t))\left[Q^{*}(t)-\phi^{\Delta}(t)\right]+\frac{\rho^{\Delta}(t)}{\rho(t)} w(t) \\
& -\frac{\alpha \rho(\sigma(t))}{r^{1 / \alpha}(t)} \frac{1}{\mathcal{K}^{\alpha}(t)}\left|\frac{w(t)}{\rho(t)}-\phi(t)\right|^{(\alpha+1) / \alpha}+\rho(\sigma(t)) \phi^{\Delta}(t)
\end{aligned}
$$

or

$$
\begin{aligned}
w^{\Delta}(t) \leq & -\rho(\sigma(t))\left[Q^{*}(t)-\phi^{\Delta}(t)\right]+\left(\rho^{\Delta}(t)\right)_{+} \phi(t) \\
& +\left(\rho^{\Delta}(t)\right)_{+}\left|\frac{w(t)}{\rho(t)}-\phi(t)\right|-\frac{\alpha \rho(\sigma(t))}{r^{1 / \alpha}(t)} \frac{1}{\mathcal{K}^{\alpha}(t)}\left|\frac{w(t)}{\rho(t)}-\phi(t)\right|^{(\alpha+1) / \alpha}, \quad t \geq t_{1} .
\end{aligned}
$$

Set $\gamma=\alpha, A=((\alpha \rho(\sigma(t))) /(r(1 / \alpha)(t)))\left(1 / \kappa^{\alpha}(t)\right), B=\left(\rho^{\Delta}(t)\right)_{+}$, and $u(t)=$ $|(w(t) / \rho(t))-\phi(t)|$ and applying Lemma 2.2 to (2.29), we have

$$
w^{\Delta}(t) \leq-\rho(\sigma(t))\left[Q^{*}(t)-\phi^{\Delta}(t)\right]+\left(\rho^{\Delta}(t)\right)_{+} \phi(t)+\frac{1}{(\alpha+1)^{\alpha+1}} \frac{r(t)\left(\rho^{\Delta}(t)\right)_{+}^{\alpha+1}}{(\rho(\sigma(t)))^{\alpha}} \kappa^{\alpha^{2}}(t) .
$$

Now integrating (2.30) from $t_{1}$ to $t$, we obtain

$$
\int_{t_{1}}^{t} \rho^{\sigma}(s)\left[Q^{*}(s)-\phi^{\Delta}(s)-\frac{\left(\rho^{\Delta}(s)\right)_{+}}{\rho^{\sigma}(s)} \phi(s)-\frac{1}{(\alpha+1)^{\alpha+1}} \frac{r(s)\left(\rho^{\Delta}(s)\right)_{+}^{\alpha+1}}{(\rho(\sigma(s)))^{\alpha+1}} \kappa^{\alpha^{2}}(s)\right] \Delta s \leq w\left(t_{1}\right),
$$

which leads to a contradiction to condition (2.18). The proof is now complete.

By different choices of $\rho(t)$ and $\phi(t)$, we obtain some sufficient conditions for the solutions of (1.6) to be oscillatory. For instance, $\rho(t)=1, \phi(t)=1$ and $\rho(t)=t, \phi(t)=1 / t$ in Theorem 2.5, we obtain the following corollaries:

Corollary 2.6. Assume that (1.7) holds. Furthermore assume that, for all sufficiently large $T$, for $T \geq t_{0}$

$$
\limsup _{t \rightarrow \infty} \int_{T}^{\infty} Q^{*}(s) \Delta s=\infty
$$

where $Q^{*}(t)$ is as in Theorem 2.5. Then every solution of (1.6) is oscillatory. 
Corollary 2.7. Assume that (1.7) holds. Furthermore assume that, for all sufficiently large $T$, for $T \geq t_{0}$

$$
\limsup _{t \rightarrow \infty} \int_{T}^{\infty}\left[\sigma(s) Q^{*}(s)-\frac{r(t)(\sigma(t))^{\alpha^{2}-\alpha}}{t^{\alpha^{2}}} \Delta s=\infty,\right.
$$

where $Q^{*}(t)$ is as in Theorem 2.5. Then every solution of (1.6) is oscillatory.

Next we establish some Philos-type oscillation criteria for (1.6).

Theorem 2.8. Assume that (1.7) holds. Suppose that there exists a function $H \in C_{\mathrm{rd}}(\mathbb{D}, \mathbb{R})$, where $\mathbb{D} \equiv\left\{(t, s) / t, s \in\left[t_{0}, \infty\right)_{\mathbb{T}}\right.$ and $\left.t>s\right\}$ such that

$$
H(t, t)=0, \quad t \geq t_{0}, \quad H(t, s) \geq 0, \quad t>s \geq 0,
$$

and $H$ has a nonpositive continuous $\Delta$-partial derivative $H^{\Delta_{s}}$ with respect to the second variable such that

$$
H^{\Delta_{s}}(\sigma(t), s)+H(\sigma(t), \sigma(s)) \frac{\rho^{\Delta}(s)}{\rho(s)}=\frac{h(t, s)}{\rho(s)}(H(\sigma(t), \sigma(s)))^{\alpha /(\alpha+1)}
$$

and for all sufficiently large $T$,

$$
\limsup _{t \rightarrow \infty} \frac{1}{H(\sigma(t), T)} \int_{T}^{t}\left[\rho^{\sigma}(s) Q^{*}(s)-\frac{(h(t, s))^{\alpha+1} r(s)}{(\alpha+1)^{\alpha+1}\left(\rho^{\sigma}(s)\right)^{\alpha}}\right] \Delta s=\infty,
$$

where $Q^{*}(t)$ is same as in Theorem 2.5. Then every solution of (1.6) is oscillatory.

Proof. We proceed as in the proof of Theorem 2.5 and define $w(t)$ by (2.20). Then $w(t)>0$ and satisfies $(2.28)$ for all $t \in\left[t_{1}, \infty\right)_{\mathbb{T}}$. Multiplying $(2.28)$ by $H(\sigma(t), \sigma(s))$ and integrating, we obtain

$$
\begin{aligned}
\int_{t_{1}}^{t} H(\sigma(t), \sigma(s)) \rho^{\sigma}(s)\left[Q^{*}(s)-\phi^{\Delta}(t)\right] \Delta s \\
\leq-\int_{t_{1}}^{t} H(\sigma(t), \sigma(s)) w^{\Delta}(s) \Delta s+\int_{t_{1}}^{t} H(\sigma(t), \sigma(s)) \frac{\rho^{\Delta}(t)}{\rho(s)} w(t) \Delta s \\
\quad-\int_{t_{1}}^{t} H(\sigma(t), \sigma(s)) \frac{\alpha \rho(\sigma(t))}{r^{1 / \alpha}(t) \rho^{(\alpha+1) / \alpha}(t)} \frac{1}{\kappa^{\alpha}(t)}\left|\frac{w(t)}{\rho(t)}-\phi(t)\right|^{(\alpha+1) / \alpha} \Delta s .
\end{aligned}
$$


Using the integration by parts formula, we have

$$
\begin{aligned}
\int_{t_{1}}^{t} H(\sigma(t), \sigma(s)) w^{\Delta}(s) \Delta s & =\left.H(t, s) w(s)\right|_{t_{1}} ^{t}-\int_{t_{1}}^{t} H^{\Delta_{s}}(\sigma(t), s) w(s) \Delta s \\
& =-H\left(t, t_{1}\right) w\left(t_{1}\right)-\int_{t_{1}}^{t} H^{\Delta_{s}}(\sigma(t), s) w(s) \Delta s
\end{aligned}
$$

Substituting (2.38) into (2.37), we obtain

$$
\begin{aligned}
\int_{t_{1}}^{t} H(\sigma(t), \sigma(s)) \rho^{\sigma}(s)\left[Q^{*}(s)-\phi^{\Delta}(t)\right] \Delta s \\
\leq H\left(t, t_{1}\right) w\left(t_{1}\right) \\
\quad+\int_{t_{1}}^{t}\left[H^{\Delta_{s}}(\sigma(t), s)+H(\sigma(t), \sigma(s)) \frac{\rho^{\Delta}(t)}{\rho(s)}\right] w(s) \Delta s \\
\quad-\int_{t_{1}}^{t} H(\sigma(t), \sigma(s)) \frac{\alpha \rho(\sigma(t))}{r^{1 / \alpha}(t) \rho^{(\alpha+1) / \alpha}(t)} \frac{1}{\mathcal{K}^{\alpha}(t)}\left|\frac{w(t)}{\rho(t)}-\phi(t)\right|^{(\alpha+1) / \alpha} \Delta s .
\end{aligned}
$$

From (2.35) and (2.39), we have

$$
\begin{aligned}
& \int_{t_{1}}^{t} H(\sigma(t), \sigma(s)) \rho^{\sigma}(s) Q(t, s) \Delta s \\
& \leq H\left(t, t_{1}\right) w\left(t_{1}\right) \\
&+\int_{t_{1}}^{t} \frac{h(t, s)}{\rho(s)} H^{\alpha /(\alpha+1)}(\sigma(t), \sigma(s)) w(s) \Delta s \\
& \quad-\int_{t_{1}}^{t} H(\sigma(t), \sigma(s)) \frac{\alpha \rho(\sigma(t))}{r^{1 / \alpha}(t) \rho^{(\alpha+1) / \alpha}(t)} \frac{1}{\kappa^{\alpha}(t)}\left|\frac{w(t)}{\rho(t)}-\phi(t)\right|^{(\alpha+1) / \alpha} \Delta s
\end{aligned}
$$

or

$$
\begin{aligned}
\int_{t_{1}}^{t} H(\sigma(t), \sigma(s)) Q(t, s) \Delta s \\
\leq H\left(t, t_{1}\right) w\left(t_{1}\right) \\
\quad+\int_{t_{1}}^{t} \frac{h(t, s)}{\rho(s)} H^{(\alpha+1) / \alpha}(\sigma(t), \sigma(s))\left|\frac{w(s)}{\rho(s)}-\phi(s)\right| \Delta s \\
\quad-\int_{t_{1}}^{t} H(\sigma(t), \sigma(s)) \frac{\alpha \rho(\sigma(t))}{r^{1 / \alpha}(t) \rho^{(\alpha+1) / \alpha}(t)} \frac{1}{\kappa^{\alpha}(t)}\left|\frac{w(t)}{\rho(t)}-\phi(t)\right|^{(\alpha+1) / \alpha} \Delta s .
\end{aligned}
$$

where $Q(t, s)=\left[\rho^{\sigma}(s) Q(t, s)-(h(t, s)) /\left(\rho(s) H^{(1 / \alpha)}(\sigma(t), \sigma(s))\right) \phi(s)\right]$. 
By setting $B=(h(t, s) / \rho(s)) H^{(\alpha+1) / \alpha}(\sigma(t), \sigma(s))$ and $A=\left((\alpha \rho(\sigma(t))) /\left(r^{1 / \alpha}(t) \rho^{(\alpha+1) / \alpha}(t)\right)\right)(1 /$ $\left.\kappa^{\alpha}(t)\right)$ in Lemma 2.2, we obtain

$$
\begin{aligned}
& \int_{t_{1}}^{t} H(\sigma(t), \sigma(s))\left[\rho^{\sigma}(s) Q(t, s)-\frac{h^{\alpha+1}(t, s) r(s) \kappa^{\alpha^{2}}(t)}{(\alpha+1)^{(\alpha+1)} \rho^{\alpha}(\sigma(s)) H(\sigma(t), \sigma(s))}\right] \Delta s \\
& \quad \leq H\left(t, t_{1}\right) w\left(t_{1}\right),
\end{aligned}
$$

which contradicts condition (2.35). This completes the proof. (1.8) holds.

Finally in this section we establish some oscillation criteria for (1.6) when the condition

Theorem 2.9. Assume that (1.8) holds and $\lim _{t \rightarrow \infty} p(t)=p<1$. Let $\left(\eta_{1}, \eta_{2}, \ldots, \eta_{n}\right)$ be $n$-tuple satisfying (2.3) of Lemma 2.1. Moreover assume that there exist positive delta differentiable functions $\rho(t)$ and $\theta(t)$ such that $\theta^{\Delta}(t) \geq 0$ and a nonnegative function $\phi(t)$ with condition (2.30) for all $t \geq t_{1}$. If

$$
\int_{t_{0}}^{\infty}\left(\frac{1}{\theta(s) r(s)} \int_{t_{0}}^{s} \theta(\sigma(v)) \bar{Q}(v) \Delta v\right)^{1 / \alpha} \Delta s=\infty
$$

where $\bar{Q}(t)=Q(t)+\sum_{i=1}^{n} Q_{i}(t)$ holds, then every solution of (1.6) either oscillates or converges to zero as $t \rightarrow \infty$.

Proof. Assume to the contrary that there is a nonoscillatory solution $x(t)$ such that $x(t)>0$, $x(\delta(t))>0, x(\tau(t))>0$, and $x\left(\tau_{i}(t)\right)>0$ for $t \in\left[t_{1}, \infty\right)_{\mathbb{T}}$ for some $t_{1} \geq t_{0}$. From Lemma 2.3 we can easily see that either $z^{\Delta}(t)>0$ eventually or $z^{\Delta}(t)<0$ eventually.

If $z^{\Delta}(t)>0$ eventually, then the proof is the same as in Theorem 2.5, and therefore we consider the case $z^{\Delta}(t)<0$.

If $z^{\Delta}(t)<0$ for sufficiently large $t$, it follows that the limit of $z(t)$ exists, say $a$. Clearly $a \geq 0$. We claim that $a=0$. Otherwise, there exists $M>0$ such that $z^{\alpha}(\tau(t)) \geq M$ and $z^{\alpha_{i}}\left(\tau_{i}(t)\right) \geq M, i=1,2, \ldots, n, t \in\left[t_{1}, \infty\right)_{\mathbb{T}}$. From (1.6) we have

$$
\left(r(t)\left(z^{\Delta}(t)\right)^{\alpha}\right)^{\Delta} \leq-M\left[Q(t)+\sum_{i=1}^{n} Q_{i}(t)\right]=-M \bar{Q}(t) .
$$

Define the supportive function

$$
u(t)=\theta(t) r(t)\left(z^{\Delta}(t)\right)^{\alpha}, \quad t \in\left[t_{1}, \infty\right)_{\mathbb{T}},
$$


and we have

$$
\begin{aligned}
u^{\Delta}(t) & =\theta^{\Delta}(t) r(t)\left(z^{\Delta}(t)\right)^{\alpha}+\theta(\sigma(t))\left(r(t)\left(z^{\Delta}(t)\right)^{\alpha}\right)^{\Delta} \\
& \leq \theta(\sigma(t))\left(r(t)\left(z^{\Delta}(t)\right)^{\alpha}\right)^{\Delta} \\
& =-M \theta(\sigma(t)) \bar{Q}(t) .
\end{aligned}
$$

Now if we integrate the last inequality from $t_{1}$ to $t$, we obtain

$$
u(t) \leq u\left(t_{1}\right)-M \int_{t_{1}}^{t} \theta(\sigma(s)) \bar{Q}(s) \Delta s
$$

or

$$
\left(z^{\Delta}(t)\right)^{\alpha} \leq-M \frac{1}{\theta(t) r(t)} \int_{t_{1}}^{t} \theta(\sigma(s)) \bar{Q}(s) \Delta s
$$

Once again integrate from $t_{1}$ to $t$ to obtain

$$
M^{1 / \alpha} \int_{t_{1}}^{t}\left(\frac{1}{\theta(s) r(s)} \int_{t_{1}}^{s} \theta(\sigma(\xi)) \bar{Q}(\xi) \Delta \xi\right)^{1 / \alpha} \Delta s \leq z\left(t_{1}\right)
$$

which contradicts condition (2.43). Therefore $\lim _{t \rightarrow \infty} z(t)=0$, and there exists a positive constant $c$ such that $z(t) \leq c$ and $x(t) \leq z(t) \leq c$. Since $x(t)$ is bounded, lim sup $\sin _{t \rightarrow \infty} x(t)=x_{1}$ and $\lim \inf _{t \rightarrow \infty} x(t)=x_{2}$. Clearly $x_{2} \leq x_{1}$. From the definition of $z(t)$, we find that $x_{1}+p x_{2} \leq$ $0 \leq x_{2}+p x_{1}$; hence $x_{1} \leq x_{2}$ and $x_{1}=x_{2}=0$. This completes proof of the theorem.

Remark 2.10. If $q_{i}(t) \equiv 0, i=1,2, \ldots, n$, or $\delta(t)=t-\delta, \tau(t)=t-\tau$, and $q_{i}(t) \equiv 0, i=1,2, \ldots, n$, then Theorem 2.5 reduces to a result obtained in [20] or [24], respecively. If $p(t) \equiv 0$, or $p(t) \equiv$ 0 , and $\alpha=1$, or $p(t) \equiv 0$, and $\tau(t)=\tau_{i}(t)=t, i=1,2, \ldots, n$, then the results established here complement to the results of $[5,9,15]$ respectively.

\section{Examples}

In this section, we illustrate the obtained results with the following examples.

Example 3.1. Consider the second order delay dynamic equation

$$
\left(x(t)+\frac{1}{t^{2}} x(\delta(t))\right)^{\Delta \Delta}+\frac{\lambda_{1}}{t^{3 / 2}} x(\sqrt{t})+\frac{\lambda_{2}}{t} x^{5 / 3}(\sqrt{t})+\frac{\lambda_{3}}{t^{2}} x^{1 / 3}(\sqrt{t})=0,
$$


for all $t \in[1, \infty)_{\mathbb{T}}$. Here $\alpha=1, \alpha_{1}=1 / 3, \alpha_{2}=5 / 3, p(t)=1 / t^{2}, q(t)=\lambda_{1} / t^{3 / 2}, q_{1}(t)=\lambda_{2} / t$, and $q_{2}(t)=\lambda_{3} / t^{2}$. Then $\eta_{1}=\eta_{2}=1 / 2$. By taking $\rho(t)=t$, and $\phi(t)=0$, we obtain

$$
\begin{aligned}
& \limsup _{t \rightarrow \infty} \int_{t_{1}}^{t} \rho^{\sigma}(s)\left[Q^{*}(s)-\frac{1}{(\alpha+1)^{\alpha+1}} \frac{r(s)\left(\rho^{\Delta}(s)\right)^{\alpha+1}}{\left(\rho^{\sigma}(s)\right)^{\alpha+1}}\right] \Delta s \\
& \quad=\limsup _{t \rightarrow \infty} \int_{t_{0}}^{t}\left[\left(\frac{\lambda_{1}}{s}\left(1-\frac{1}{s}\right)+\frac{\sqrt{\lambda_{2} \lambda_{3}}}{s}\left(1-\frac{1}{s}\right)\right)-\frac{1}{4 \sigma(s)}\right] \Delta s \\
& \quad \geq \limsup _{t \rightarrow \infty}^{t} \int_{t_{0}}^{t}\left(\left[\lambda_{1}+\sqrt{\lambda_{2} \lambda_{3}}-\frac{1}{4}\right] \frac{1}{s}-\frac{\lambda_{1}+\sqrt{\lambda_{2} \lambda_{3}}}{s^{2}}\right) \Delta s \\
& \quad \rightarrow \infty \text { if } \lambda_{1}+\sqrt{\lambda_{2} \lambda_{3}}>1 / 4 .
\end{aligned}
$$

By Theorem 2.5, all solutions of (3.1) are oscillatory if $\lambda_{1}+\sqrt{\lambda_{2} \lambda_{3}}>1 / 4$.

Example 3.2. Consider the second order neutral delay dynamic equation

$$
\left(\left(\left(x(t)+\frac{1}{2} x(\delta(t))\right)^{\Delta}\right)^{3}\right)^{\Delta}+\frac{\sigma^{3}(t)}{t^{4}} x^{3}\left(\frac{t}{2}\right)+\frac{\sigma(t)}{t^{2}} x^{5}\left(\frac{t}{3}\right)+\frac{\sigma(t)}{t^{2}} x^{1 / 3}\left(\frac{t}{3}\right)=0
$$

for all $t \in[1, \infty)_{\mathbb{T}}$. Here $r(t)=1, p(t)=1 / 2, q(t)=\left(\sigma^{3}(t)\right) / t^{4}, \tau(t)=t / 2, \tau_{1}(t)=\tau_{2}(t)=t / 3$, $\alpha=3, \alpha_{1}=5, \alpha_{2}=1 / 3$. From Corollary 2.6, every solution of (3.3) is oscillatory.

\section{Acknowledgment}

The authors thank the referees for their constructive suggestions and corrections which improved the content of the paper.

\section{References}

[1] M. Bohner and A. Peterson, Dynamic Equations on Time Scales: An Introduction with Applications, Birkhäuser, Boston, Mass, USA, 2001.

[2] M. Bohner and A. Peterson, Eds., Advances in Dynamic Equations on Time Scales, Birkhäuser Boston Inc., Boston, Mass, USA, 2003.

[3] S. Hilger, "Analysis on measure chains-a unified approach to continuous and discrete calculus," Results in Mathematics, vol. 18, no. 1-2, pp. 18-56, 1990.

[4] R. Agarwal, M. Bohner, D. O'Regan, and A. Peterson, "Dynamic equations on time scales: a survey," Journal of Computational and Applied Mathematics, vol. 141, no. 1-2, pp. 1-26, 2002.

[5] R. P. Agarwal, D. O’Regan, and S. H. Saker, "Oscillation criteria for second-order nonlinear neutral delay dynamic equations," Journal of Mathematical Analysis and Applications, vol. 300, no. 1, pp. 203 217, 2004.

[6] L. Erbe, A. Peterson, and S. H. Saker, "Oscillation criteria for second-order nonlinear delay dynamic equations," Journal of Mathematical Analysis and Applications, vol. 333, no. 1, pp. 505-522, 2007.

[7] S. H. Saker, D. O’Regan, and R. P. Agarwal, "Oscillation theorems for second-order nonlinear neutral delay dynamic equations on time scales," Acta Mathematica Sinica, vol. 24, no. 9, pp. 1409-1432, 2008. 
[8] J. Shao and F. Meng, "Oscillation theorems for second-order forced neutral nonlinear differential equations with delayed argument," International Journal of Differential Equations, vol. 2010, Article ID 181784, 15 pages, 2010.

[9] R. P. Agarwal, D. R. Anderson, and A. Zafer, "Interval oscillation criteria for second-order forced delay dynamic equations with mixed nonlinearities," Computers \& Mathematics with Applications, vol. 59, no. 2, pp. 977-993, 2010.

[10] R. P. Agarwal and A. Zafer, "Oscillation criteria for second-order forced dynamic equations with mixed nonlinearities," Advances in Difference Equations, vol. 2009, Article ID 938706, 20 pages, 2009.

[11] C. Li and S. Chen, "Oscillation of second-order functional differential equations with mixed nonlinearities and oscillatory potentials," Applied Mathematics and Computation, vol. 210, no. 2, pp. 504-507, 2009.

[12] S. Murugadass, E. Thandapani, and S. Pinelas, "Oscillation criteria for forced second-order mixed type quasilinear delay differential equations," Electronic Journal of Differential Equations, p. No. 73, 9, 2010.

[13] Y. G. Sun and F. W. Meng, "Oscillation of second-order delay differential equations with mixed nonlinearities," Applied Mathematics and Computation, vol. 207, no. 1, pp. 135-139, 2009.

[14] Y. G. Sun and J. S. W. Wong, "Oscillation criteria for second order forced ordinary differential equations with mixed nonlinearities," Journal of Mathematical Analysis and Applications, vol. 334, no. 1, pp. 549-560, 2007.

[15] M. Ünal and A. Zafer, "Oscillation of second-order mixed-nonlinear delay dynamic equations," Advances in Difference Equations, vol. 2010, Article ID 389109, 21 pages, 2010.

[16] Z. Zheng, X. Wang, and H. Han, “Oscillation criteria for forced second order differential equations with mixed nonlinearities," Applied Mathematics Letters, vol. 22, no. 7, pp. 1096-1101, 2009.

[17] A. K. Tripathy, "Some oscillation results for second order nonlinear dynamic equations of neutral type," Nonlinear Analysis: Theory, Methods \& Applications, vol. 71, no. 12, pp. e1727-e1735, 2009.

[18] S. H. Saker, R. P. Agarwal, and D. O’Regan, “Oscillation results for second-order nonlinear neutral delay dynamic equations on time scales," Applicable Analysis, vol. 86, no. 1, pp. 1-17, 2007.

[19] Y. Şahíner, "Oscillation of second-order neutral delay and mixed-type dynamic equations on time scales," Advances in Difference Equations, vol. 2006, Article ID 65626, 9 pages, 2006.

[20] H.-W. Wu, R.-K. Zhuang, and R. M. Mathsen, "Oscillation criteria for second-order nonlinear neutral variable delay dynamic equations," Applied Mathematics and Computation, vol. 178, no. 2, pp. 321-331, 2006.

[21] Y. Sun, Z. Han, T. Li, and G. Zhang, "Oscillation criteria for second-order quasilinear neutral delay dynamic equations on time scales," Advances in Difference Equations, vol. 2010, Article ID 512437, 14 pages, 2010.

[22] Z. Han, S. Sun, T. Li, and C. Zhang, "Oscillatory behavior of quasilinear neutral delay dynamic equations on time scales," Advances in Difference Equations, vol. 2010, Article ID 450264, 24 pages, 2010.

[23] G. H. Hardy, J. E. Littlewood, and G. Pólya, Inequalities, Cambridge University Press, Cambridge, UK, 2nd edition, 1952.

[24] S. H. Saker, "Oscillation of second-order nonlinear neutral delay dynamic equations on time scales," Journal of Computational and Applied Mathematics, vol. 187, no. 2, pp. 123-141, 2006. 